\section{Charged projectile spectrometry using solid-state nuclear track detector
of the PM-355 type using solid-state nuclear track detector
of the PM-355 type} (n)

\author{
Aneta Malinowska, \\ Marian Jaskóła, \\ Andrzej Korman, \\ Adam Szydłowski, \\ Karol Malinowski, \\ Mirosław Kuk
}

\begin{abstract}
$\overline{\text { Abstract. To use effectively any radiation detector in high-temperature plasma experiments, it must have a lot }}$ of benefits and fulfill a number of requirements. The most important are: a high energy resolution, linearity over a wide range of recorded particle energy, high detection efficiency for these particles, a long lifetime and resistance to harsh conditions existing in plasma experiments and so on. Solid-state nuclear track detectors have been used in our laboratory in plasma experiments for many years, but recently we have made an attempt to use these detectors in spectroscopic measurements performed on some plasma facilities. This paper presents a method that we used to elaborate etched track diameters to evaluate the incident projectile energy magnitude. The method is based on the data obtained from a semiautomatic track scanning system that selects tracks according to two parameters, track diameter and its mean gray level.
\end{abstract}

Key words: solid-state nuclear track detectors (SSNTDs) • PM-355 • energy resolution of proton and deuteron - calibration diagrams • track mean gray level

A. Malinowska ${ }^{\bowtie}$, M. Jaskóła, A. Korman,

A. Szydłowski, M. Kuk

National Centre for Nuclear Research (NCBJ),

7 Andrzeja Soltana Str., 05-400 Otwock/Swierk, Poland,

Tel.: +48 22718 0417, Fax: +48 22779 3481,

E-mail: aneta.malinowska@ncbj.gov.pl

\section{K. Malinowski}

Institute of Plasma Physics and Laser Microfusion, 23 Hery Str., 01-497 Warsaw, Poland and National Centre for Nuclear Research (NCBJ), 7 Andrzeja Soltana Str., 05-400 Otwock/Swierk, Poland

Received: 21 October 2014

Accepted: 20 May 2015

\section{Introduction}

Starting from the pioneering work of Silk and Barnes the application of solid-state nuclear detectors (SSNTDs) has grown considerably [1]. After the discovery by Cartwright et al. of the CR-39 plastic material, the role of this kind of track detector has rapidly increased [2]. Owing to its original and extraordinary properties and many advantages over other track detectors (such as mica, quartz, cellulose nitrate), the CR-39 material has found many applications in different disciplines of science and technology [3].

Some properties of SSNTDs, such as their high sensitivity to ions over a wide energy range, their nearly $100 \%$ detection efficiency for charged projectiles, resistance to different environments, low production and running costs, small sensitivity to photons and electrons, make them very useful diagnostic tools for thermonuclear plasma experiments. Therefore, one can observe an increasing interest in such detectors in many plasma laboratories [4-7].

In the literature, many papers have been devoted to studies of the energy resolution of different radiation detectors. Such studies can also be found in many monographs, e.g., the book by England [8]. Unfortunately, papers concerning the energy resolution of plastic SSNTDs are mainly devoted to $\alpha$-particle spectroscopy. In 1974 Lück developed the technique of etching the detector sheets from the reverse side to the incident projectile $[9,10]$. The 
method of particle energy determination is based on the end-point of the particle range measurements. In this method, it is best if the detector sheets have a thickness slightly greater than the particle range.

In the 1980 s, a group of scientists from the Physics Laboratory of the University of Bristol, England, developed a technique based on the commercially available CR-39 plastic material, which demonstrates a high resolution, especially in $\alpha$-particle spectroscopy $[11,12]$. Automated scanning measurements of the track parameters developed by this group and computer track profile image analysis of track structure greatly extended the applications of plastic track detectors. Automated image analysis systems can be used to measure up to a few thousand tracks per hour, whereas an experienced scanner can manually measure only a few hundred tracks per week. The method proposed by the University of Bristol group relies on the determination of track length in the plastic material. It is usually made using the measured etch track geometrical parameters. One of the proposed methods is based on the three horizontally measurable parameters (minor axis, overall track length and the diameter of the etched out end) and the authors demonstrated that this method leads to good accuracy in the length measurement and consequently to improved $\alpha$-particle energy resolution.

In the papers by Santos et al. and Immè et al. a method is presented that analyzes the diameters of the $\alpha$-particle etch pits and the optical density of the detector called the 'track gray level' $[13,14]$. This analysis can give information on the track depth that increases monotonically with $\alpha$-particle energy and its angle of incidence. A suitable analysis routine carried out by means of a semiautomatic system using this method allows the identification of tracks according to projectile energy.

In this work, we used the results obtained by our group in the period 2000-2014. These results were obtained using commercial PM-355 material and we partly published them in our previous publications [15-20]. In this paper, we report on a track analysis performed by a semiautomatic system that selected tracks according to two parameters, diameter of the etched pits and mean gray level. Combining the information on the two parameters (track diameter and mean gray level) allowed us to distinguish well the incident proton and deuteron energy values. A series of measurements was carried out by our group over several years at the Institute for Nuclear Studies (Swierk). The main aim of these measurements was to observe changes of track dimensions as a function of projectile energy and the etching time. During the period 2000-2014, we collected a large number of data, which were later utilized in many high-temperature plasma experiments. In plasma physics diagnostics, it may often be necessary to select tracks according to the recorded projectile type and its energy. For example, to measure $3 \mathrm{MeV}$ fusion reaction protons emitted from the PF-360 and PF-1000 plasma focus facilities, we had to distinguish these products from primary ions $[4,21]$. The same problem appeared in experiments performed at the TEXTOR tokamak (Juelich, Germany), where to measure fusion reaction protons we had to cut low energy primary ions $[6,21]$. In experiments conducted at LULI (Palaiseau, France) to measure energy spectra of fast protons emitted from laser-produced plasma, we used a few detector samples that were masked with $\mathrm{Al}$ foils of different thicknesses [7, 21]. To distinguish craters induced by protons from craters produced by other ion species, we relied on the earlier determined calibration diagrams.

\section{Experiment}

In this paper, some rectangular samples (cut into about $1 \times 3 \mathrm{~cm}^{2}$ pieces) with a thickness of $0.5 \mathrm{~mm}$ of the PM-355 type detector were irradiated with light ions $\left(\mathrm{H}^{+}\right.$- and $\mathrm{D}^{+}$-ions) of different energies provided by various particle accelerators, but mainly by the LECH accelerator (Van de Graaff type) [16-21]. These samples were prepared from the detector batch bought from the Page Pershore Moulding Company in 2009 [15]. During the irradiations, ion energy values were changed in steps of 200 or $300 \mathrm{keV}$ in the energy interval from $200 \mathrm{keV}$ to $2 \mathrm{MeV}$. The irradiation was performed in a vacuum scattering chamber at almost normal incidence on the SSNTD samples by ions elastically scattered from suitably thin target foils. The samples' irradiation time was approx. 10-15 min. A self-supporting carbon foil (about $10 \mu \mathrm{g} / \mathrm{cm}^{2}$ thick) and gold foil (about $100 \mu \mathrm{g} / \mathrm{cm}^{2}$ thick) were used as a scatterer. The ion fluxes were measured using a Si surface barrier detector in order to measure the energy spectra of the scattered ions. The fluxes of the projectiles that hit the track detector samples did not exceeded (2-4) $\times 10^{4}$ particles $/ \mathrm{cm}^{2}$. The energy spread of the elastically scattered projectiles depended mainly on the geometrical conditions and the total energy loss in the target material and ranged from about $30 \mathrm{keV}$ (for $200 \mathrm{keV}$ protons) to $20 \mathrm{keV}$ (for $2 \mathrm{MeV}$ protons). For deuterons, these values were almost 1.3 times higher.

After irradiation, the samples were chemically etched in steps in a $6.25 \mathrm{~N}$ water solution of $\mathrm{NaOH}$ at a temperature of $70 \pm 1{ }^{\circ} \mathrm{C}$ during selected time intervals. The etching procedure was interrupted every $2 \mathrm{~h}$. Track parameters were measured under a Nikon type (Japan) optical microscope. A total magnification of 1000 was used to determine track diameter magnitudes vs. projectiles energies and etching time $t$. The track detector readout was made using a semiautomatic system composed of the optical microscope connected to a PC by means of a CCD camera (Nikon DS.-Fi2, 5.24 Mpix) and suitable software (Nikon - NIS - Elements BR 4.00.03 - 64 bit). Etched tracks were counted automatically in 10 different fields taken randomly surrounding the center of the detector. The etched tracks appeared as dark spots on a bright background. Because the angle of incidence of the projectiles reaching the detector surface was almost perpendicular, we observed that the shape of the etch pits was almost circular. To analyze the etching tracks, we used another program, ImageJ. This image processing program was developed at the National Institutes of 
Health [22]. Microscope image processing is a broad term that covers the use of digital image processing techniques to analyze and present images obtained from a microscope. A number of manufacturers of microscopes now specifically design and equip their products with features that allow the user to interface the microscope to an image processing system. This image processing reproduces the information contained in the examined sample. This might include adjusting the brightness and contrast of the image, averaging images to reduce image noise and correcting for illumination non-uniformities.

\section{Results and discussion}

Some results of our detailed calibration measurements are presented in Fig. 1. The figure shows the proton and deuteron track diameters $D(E, t)$ expressed as a function of projectile energy and etching time. One can see that the track diameter increase very fast in the region of relatively low particle energies. When the maxima are obtained, the track diameters decrease monotonically with a further increase of projectile energy. In the $D(E, t)$ decreasing region of $\mathrm{D}(\mathrm{E})$ vs. projectile energy $\mathrm{E}$, the $D(E, t)$ dependences approximately reflect the stopping power curve (i.e. $\mathrm{dE} / \mathrm{dx}$ vs. projectile energy dependence). Generally the variation of the track diameter diagram vs. particle energy is similar to the behavior of the Bragg curve. One can also conclude that the deuteron track diameters D are generally higher than the proton track diameters for the same projectile energies. The data presented in Fig. 1 also indicate that tracks of the same diameter can be produced by projectiles of different incident energies, which makes it difficult to determine unambiguously the particle energy. The etch pits produced by protons or deuterons with energies above those at which the maxima occur may have diameters equal to craters produced by projectiles with energies below maximum. Such behavior of the track diameter $\mathrm{D}(\mathrm{E}, \mathrm{t})$ vs. projectile energy and etching time is presented by a non-monotonic function and is a fundamental limitation of this method for

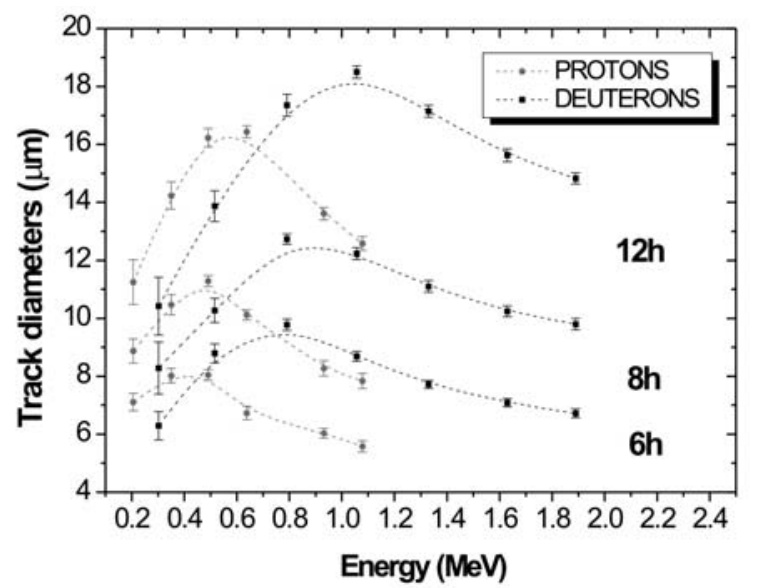

Fig. 1. Evolution of track opening diameters $\mathrm{D}$ for protons and deuterons as a function of incident projectile energy and etching times. Lines are drawn to guide the eye.

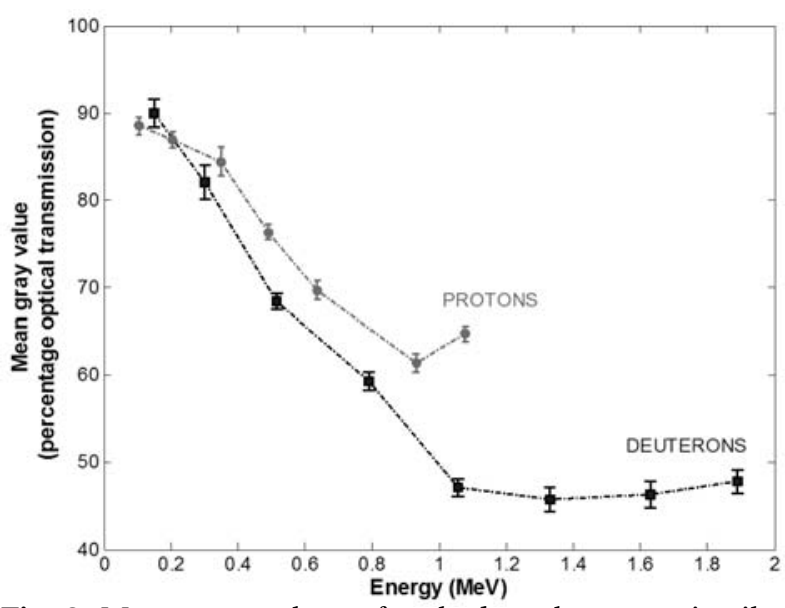

Fig. 2. Mean gray values of etched tracks vs. projectiles incident energy for protons and deuterons. Lines are drawn to guide the eye.

practical (spectroscopic) applications over a wide range of projectile energy.

Taking into consideration this heterogeneity one can conclude that measurement of track diameter is not sufficient to determine the projectile energy. In order to overcome this ambiguity, we propose to perform additionally an analysis of the track end grayness to estimate the so-called track mean gray level. The method of mean gray level measurements is illustrated in the paper by Immè et al. for tracks induced by $\alpha$ particles [14]. In Figs. 2 and $3(a, b)$, the distributions of the mean gray level magnitudes
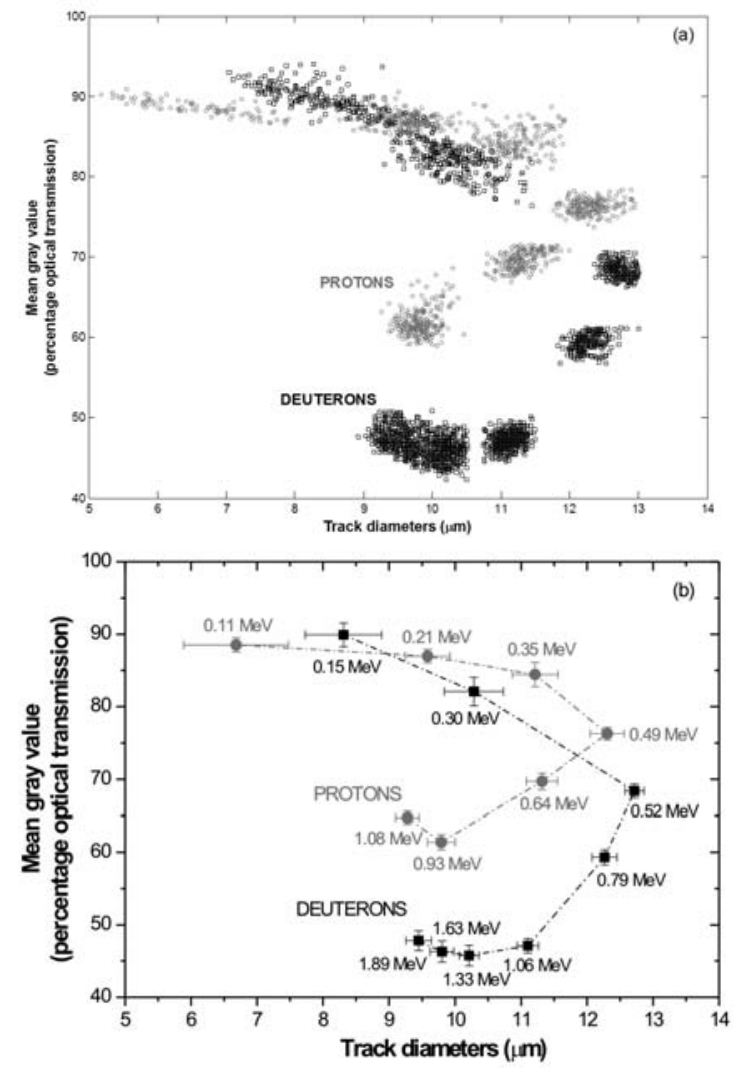

Fig. 3. Two-dimensional distribution of track mean gray value vs. track diameters D (a) and for mean gray value of track diameters D (b) for $8 \mathrm{~h}$ of etching time and different energies of projectiles group for protons and deuterons. Lines are drawn to guide the eye. 
obtained for proton and deuteron tracks are presented in two forms. In the first form, the mean gray level of craters is presented vs. proton and deuteron energies (Fig. 2). Whereas in the second two-dimensional form, the mean gray level is plotted vs. track diameter, Fig. $3(\mathrm{a}, \mathrm{b})$, for different hydrogen projectile energies (the energy values are given in Fig. 3(b)). An examination of Figs. 2 and 3 indicates that the mean gray level dependences presented, both vs. projectile energy and vs. track diameter, shows similar behavior for protons and deuterons. It is seen that protons or deuterons of higher energy give tracks of lower mean gray level and for energies above $1 \mathrm{MeV}$, a saturation effect occurs. Therefore, using the data from both the track diameter diagrams (i.e. as presented in Fig. 1) and from the mean gray level dependences (i.e. those shown in Figs. 2 and $3(a, b)$ ), we are able to approximate roughly the projectile energy. Moreover, we are in a position to distinguish lower energy projectiles from higher energy ones, which produce craters of the same diameters but of different mean gray level.

Histograms presenting diameters of craters induced in the PM-355 detector by protons and deuterons vs. energy of these projectiles and detec-
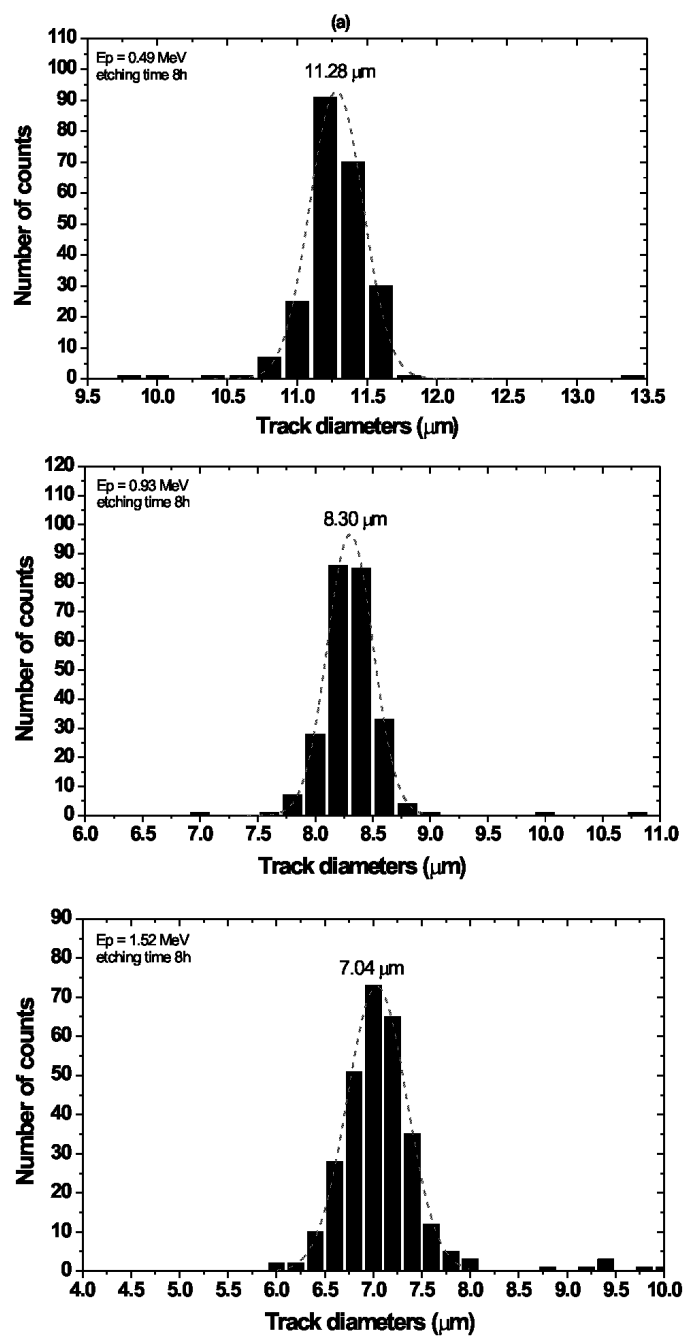

tor etching time are presented in Fig. 4(a,b) and were fitted by suitable gaussian curves. The full width at half maximum (FWHM) values of these distributions demonstrates the energy resolution of the projectile energy determination, which is based only on the track diameter measurement. The width demonstrates the practical measurement resolution achievable by the system on the etched tracks using only one parameter in form of the track diameter D. The spread of the FWHM values is in the range $0.17-0.5 \mu \mathrm{m}$ and does not depend on the etching time. The track parameters such as their diameters and lengths measured by other groups on samples of CR-39 material are similar to our values, for example, the University of Bristol group measured track lengths with an accuracy of $0.2 \mu \mathrm{m}[11,12]$.

The FWHM values expressed in megaelectron volts are presented in Fig. 5 as a function of proton and deuteron energy. The transformation of the track dimension unit, micrometer, into the projectile energy unit, megaelectron volts, was made using the calibration diagrams presented in Fig. 1. Examining the obtained FWHM (E) dependence (Fig. 5), one can see that the lowest values, that is, the higher resolution, are in the region $0.2-0.5 \mathrm{MeV}$, that is, where the slope of the calibration diagrams is the
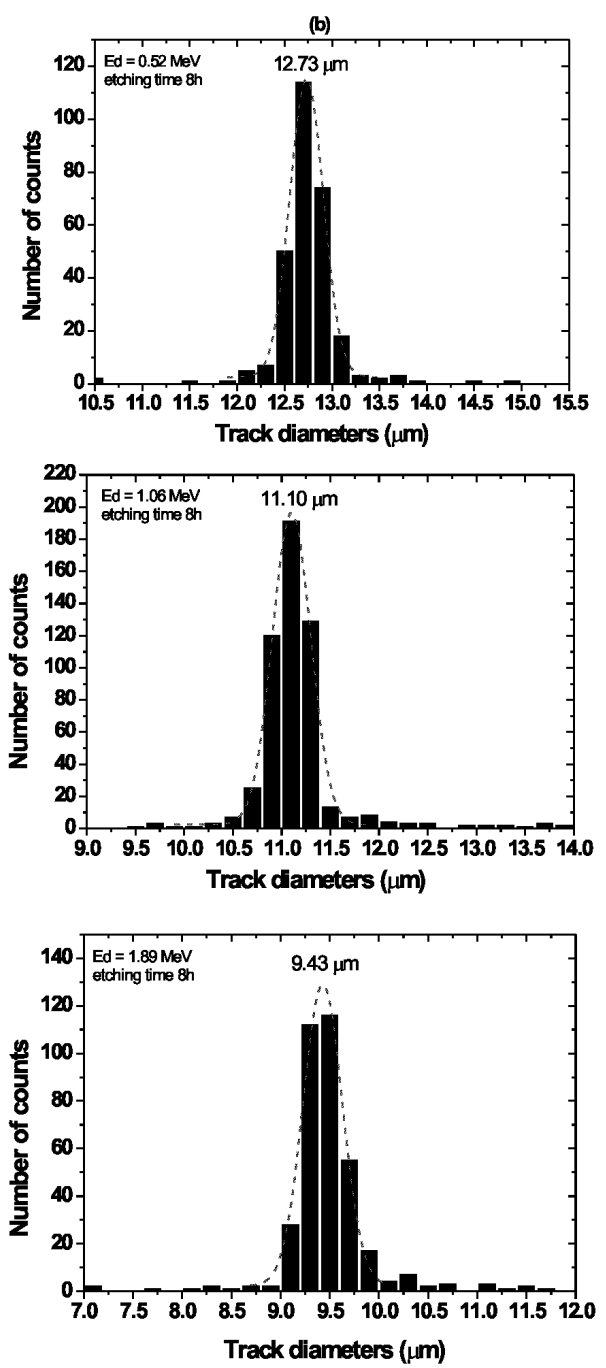

Fig. 4. Histograms of the measured distribution of track diameter D for $8 \mathrm{~h}$ etching time for different projectile energies (for protons (a) and deuterons (b)). The broken lines represent the gaussian distribution. 


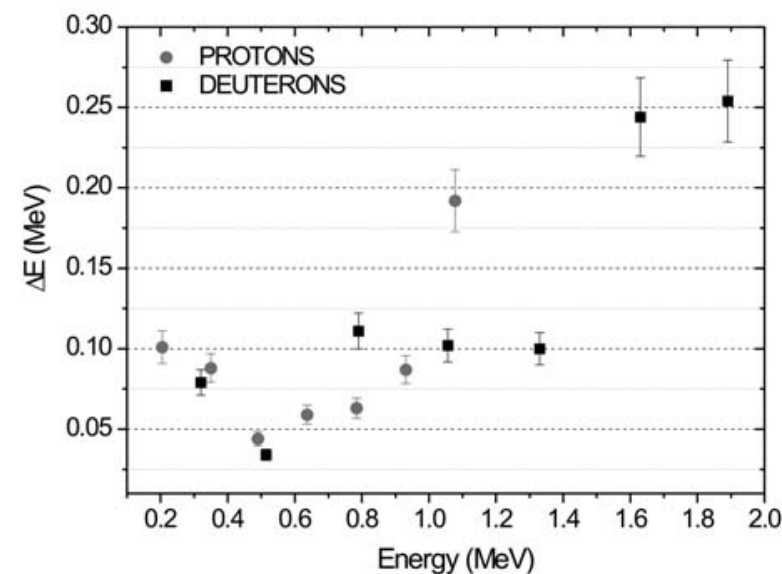

Fig. 5. The energy resolution of the SSNTD as determined for proton and deuteron projectiles and presented vs. their energy.

highest (Fig. 1). In the region where the slope of the $\mathrm{D}(\mathrm{E}, \mathrm{t})$ dependence is smaller (the curve is more flat), the energy resolution is worse. Generally, the energy resolution FWHM of the SSNTDs tested in this work is influenced by different factors, like variation of the $V_{B}$ values, even if the same detector material as well as etching conditions (temperature, $\mathrm{NaOH}$ concentration and duration of etching time) are used, and also by differences associated with the PM-355 material production procedure $\left(V_{B}\right)[18$, 19]. Also important is the selection of appropriate input parameters (like circularity, size, threshold etc.) for the program dealing with the graphics processing.

Examination of the track diameters presented in Fig. 1 vs. particle energy and etching time is not the best method to estimate the projectile energy with a good energy resolution. The degeneracy of the $\mathrm{D}(\mathrm{E}, \mathrm{t})$ calibration curve makes it impossible as was shown by different researchers. The better method is the application of track length measurements as shown in papers published by the Bristol University group and the Dresden University of Technology (Germany) group [11, 12, 23].

\section{Summary and conclusions}

The most important results reported in this paper can be summarized as follows:

1. A semiautomatic track analyzing system is used in our laboratory for scanning SSNTD samples applied in different thermonuclear plasma diagnostic tools and irradiated with protons, deuterons, $\alpha$ particles and other ion species. When this image analysis system was used for scanning detectors that were irradiated with hydrogen ions, it provided very quickly, and with a high accuracy, such etched pit parameters as track diameter and track mean gray level.

2. Measurements only of the track diameter or the track mean gray level do not give enough information to determine the energy of the incident charged projectiles, but using both these parameters makes it possible to approximate roughly the proton and deuteron energy. In some practical situation, to better distinguish craters induced by protons from craters produced by other projectiles, it is very useful to apply absorbers with different thickness to mask partially the detector sample surface.

Acknowledgments. We would like to thank Dr N. Keeley for a critical reading of the manuscript and for constructive suggestions.

\section{References}

1. Silk, E. C. H., \& Barnes, R. S. (1959). Examination of fission fragment tracks with an electron microscope. Philos. Mag., 4, 970-972.

2. Cartwright, B. G., Shirk, E. K., \& Price, P. B. (1978). A nuclear track recording polymer of unique sensitivity and resolution. Nucl. Instrum. Methods, 153, 457-460.

3. Durrani, S. A., \& Bull, R. K. (1987). Solid state nuclear track detection. New York: Pergamon Press.

4. Malinowska, A., Szydłowski, A., Sadowski, M. J., Żebrowski, J., Scholz, M., Paduch, M., Jaskóła, M., \& Korman, A. (2008). Measurements of fusion-produced protons by means of SSNTDs. Radiat. Meas., 43, S295-S299.

5. Sinenian, N., Rosenberg, M. J., Manuel, M., McDuffee, S. C., Casey, D. T., Zylstra, A. B., Rinderknecht, H. G., Gatu Johnson, M., Séguin, F. H., Frenje, J. A., Li, C. K., \& Petrasso, R. D. (2011). The response of CR-39 nuclear track detector to 1-9 MeV protons. Rev. Sci. Instrum., 82, 103303.

6. Szydłowski, A., Malinowska, A., Sadowski, M. J., Jaskóła, M., Korman, A., Wan Wassenhove, G., Bonheure, G., Schweer, B., and TEXTOR Team, Gałkowski, A., \& Małek, K. (2008). Measurement of fusion-reaction protons in TEXTOR tokamak plasma by means of solid-state nuclear track detectors of the CR-39/PM-355 type. Radiat. Meas., 43, S290-S294.

7. Szydłowski, A., Badziak, J., Fuchs, J., Kubkowska, M., Parys, P., Rosiński, M., Suchańska, R., Wołowski, J., Antici, P., \& Mancic, A. (2009). Application of solid-state nuclear track detectors of the CR-39/ PM-355 type for measurements of energetic protons emitted from plasma produced by an ultra-intense laser. Radiat. Meas., 44, 881-885.

8. England, J. B. A. (1974). Techniques in nuclear structure. New York: Wiley-Macmillan Press.

9. Lück, H. B. (1974). Energy spectrometry of protons by means of a dielectric track detector. Nucl. Instrum. Methods, 119, 403-404.

10. Lück, H. B. (1975). Investigations on energy resolution in detecting alpha particles using a cellulose nitrate detector. Nucl. Instrum. Methods, 124, 359-363.

11. Fews, A. P. (1992). Fully automated image analysis of etched tracks in CR-39. Nucl. Instrum. Methods Phys. Res. Sect. B-Beam Interact. Mater. Atoms, 71, 465-478.

12. Fews, A. P. (1992). Flexible analysis of etched nuclear particle tracks. Nucl. Instrum. Methods Phys. Res. Sect. B-Beam Interact. Mater. Atoms, 72, 91-103.

13. Santos, N. F., Iunes, P. J., Paulo, S. R., Guedes, S., \& Hadler, J. C. (2010). CR-39 alpha particle spectrometry for the separation of the radon decay product ${ }^{214}$ Po from the thoron decay product ${ }^{212}$ Po. Radiat. Meas., 45, 823-826.

14. Immè, G., Morelli, D., Aranzulla, M., Catalano, R., \& Mangano, G. (2013). Nuclear track detector char- 
acterization for alpha-particle spectroscopy. Radiat. Meas., 50, 253-257.

15. Catalog Page Pershore Moulding Company. (1998).

16. Szydłowski, A., Banaszak, A., Czyżewski, T., Fijał, I., Jaskóła, M., Korman, A., Sadowski, M., \& Kretschmer, W. (2001). Advantage of PM-355 nuclear track detector in light-ion registration and high temperature plasma diagnostics. Radiat. Meas., 34(1/6), 325-329.

17. Szydłowski, A., Sartowska, B., Banaszak, A., Choiński, J., Fijał, I., Jaskóła, M., Korman, A., \& Sadowski, M. J. (2005). Calibration of PM-355 nuclear track detectors, comparison of track diameter diagrams with track depth characteristics. Radiat. Meas., 40, 401-405.

18. Malinowska, A., Szydłowski, A., Jaskóła, M., Korman, A., Malinowski, K., \& Kuk, M. (2012). Calibration of new batches and a study of applications of nuclear track detectors under the harsh conditions of nuclear fusion experiments. Nucl. Instrum. Methods Phys. Res. Sect. B-Beam Interact. Mater. Atoms, 281, 56-60.
19. Malinowska, A., Szydłowski, A., Jaskóła, M., Korman, A., Sartowska, B., Kuehn, T., \& Kuk, M. (2013). Influence of high temperature on solid state nuclear track detector parameters. Rev. Sci. Instrum., 84, 073517-073521.

20. Szydłowski, A., Malinowska, A., Jaskóła, M., Korman, A., Malinowski, K., \& Kuk, M. (2013). Calibration studies of the application of nuclear track detectors to the detection of charged particles. Radiat. Meas., 50, 258-262.

21. Malinowska, A., Szydłowski, A., Jaskóła, M., Korman, A., Żebrowski, J., Sartowska, B., Sadowski, M. J., \& Badziak, J. (2008). Calibration and application of modern track detectors CR-39/PM355 in nuclear physics and high temperature plasma experiments. Nukleonika, 53(Suppl. 2), S15-S19.

22. Collins, T. J. (2007). ImageJ for microscopy. BioTechniques, 43(Suppl. 1), 25.

23. Dörschel, B., Bretschneiderm, R., Hermsdorf, D., Kadner, K., \& Kühne, H. (1999). Measurement of the track etch rates along proton and alpha particle trajectories in CR-39 and calculation of the detection efficiency. Radiat. Meas., 31, 103-107. 